\title{
Superhydrophilic surfaces from short and medium chain solvo-surfactants
}

\author{
Romain VALENTIN \\ Zéphirin MOULOUNGUI \\ Université de Toulouse, \\ INP-ENSIACET, \\ LCA (Laboratoire de Chimie Agro- \\ industrielle), \\ INRA, \\ UMR $1010 \mathrm{CAl}$, \\ F-31030 Toulouse, \\ France \\ $<$ romain.valentin@ensiacet.fr $>$
}

Article received 8 November 2012

Accepted 15 November 2012

\begin{abstract}
Pure monoglycerides (GM-CS) and glycerol carbonate esters (GCE-CS) are two families of oleochemical molecules composed of a polar part, glycerol for GM-Cs, glycerol carbonate for GCE-Cs, and a fatty acid lipophilic part.

From a chemical point of view, GM-Cs include two free oxygen atoms in the hydroxyl functions and one ester function between the fatty acid and the glycerol parts. GCE-CS contain two blocked oxygen atoms in the cyclic carbonate backbone and three esters functions: two endocyclic in the five-membered cyclic carbonate function, one exocyclic between the fatty acid and glycerol carbonate parts. At the physico-chemical level, GMCs and GCE-Cs are multifunctional molecules with amphiphilic structures: a common hydrophobic chain to the both families and a polar head, glycerol for GMs and glycerol carbonate for GCE-CS. Physicochemical properties depend on chain lengths, odd or even carbon numbers on the chain, and glyceryl or cyclocarbonic polar heads.

The solvo-surfactant character of GM-Cs and overall GCE-Cs were discussed through the measurements of critical micellar concentration (CMC) or critical aggregation concentration (CAC). These surface active glycerol esters/glycerol carbonate esters were classified following their hydrophilic/hydrophobic character correlated to their chain length (LogP octanol/water $=f($ atom carbon number)). Differential scanning calorimetry and optical polarized light microscopy allow us to highlight the selfassembling properties of the glycerol carbonate esters alone and in presence of water. We studied by thermal analysis the polymorphic behaviour of GCE-CS, and the correlation between their melting points versus the chain lengths.

Coupling the self-aggregation and crystallization properties, superhydrophilic surfaces were obtained by formulating GM-Cs and GCE-Cs. An efficient durable water-repellent coating of various metallic and polymeric surfaces was allowed. Such surfaces coated by self-assembled fatty acid esters in a stable coagel state present a novel solution for the water-repellent coating of surfaces.
\end{abstract}

Key words: pure monoglycerides, pure glycerol carbonate esters, formulation, solvosurfactant, critical aggregation concentration, self-assembling, superhydrophilicity, $p$ olymorphism

of some of the applications that could be enabled by the creation of surfaces that either completely resist wetting by water (superhydrophobic state) or that are completely and instantaneously wet by water (high hydrophilic and superhydrophilic states). Superhydrophilicity and superwetting surfaces refer to textured and/or structured materials (rough and/or porous) on which water (liquid) spreads completely (Drelich and
Chibowski, 2010). To obtain such surfaces, two basic approaches have been reported (Cebeci et al., 2006). The first involves the use of photochemically active materials such as $\mathrm{TiO}_{2}$ that become superhydrophilic after exposure to UV, or with suitable chemical modifications, visible radiation. Surface coatings based on $\mathrm{TiO}_{2}$ typically lose their superhydrophilic qualities within minutes to hours when placed in a dark

To cite this article: Valentin R, Mouloungui Z. Superhydrophilic surfaces from short and medium chain solvo-surfactants. OCL 2013; 20(1): 33-44. doi : 10.1684/ocl.2012.0490 
environment, although much progress has been made toward eliminating this potential limitation. The second case involves the use of textured surfaces to promote superwetting behavior. In nature, superhydrophilicity has been developed by different organs of plants and is caused by different micro- and nanostructures (Koch and Barthlott, 2009). Superhydrophilic plant surfaces can be divided into those that are permanently wet, absorb water over their surfaces and let water spread over the surface. In the last case, water droplets spread rapidly on the peristome and form thin films, which make the peristome extremely slippery for insects (Bauer et al., 2008). Fast water spreading was observed on the leaves plants where glands secrete hydrophilic substances like saponins that, in combination with the surface roughness, lead to the superhydrophilicity. Water spreading is favored when surface roughness presents an arrangement where capillarity phenomena occur (Koch and Barthlott, 2009). The superhydrophilicity provides a faster evaporation of the water by an increase of the water-air interface. Thus, water evaporates from a superhydrophilic surface much faster than that from a hydrophilic or superhydrophobic one, where water forms semi-spherical or spherical droplets. Here we showed how surface-active molecules such as fatty acid esters respond adequately to the question of water-retaining by polymorphic liquid crystalline phases combining order and mobility at a molecular and supramolecular level. Therefore, glycerol fatty acid esters "monoglycerides" and "glycerol carbonate fatty acid esters" are of great importance. They are solvo-surfactants and show self-assembly phenomena if their concentration is higher than a certain critical aggregation concentration (CAC). There are some examples of self-assembly structures formed by polar lipids including glycerol fatty acid esters (Leser et al., 2006). Carbonic glycerylic fatty acid esters or GCE-Cs present a locked glycerol polar head. Pure GM-Cs are associated with variable lengths of hydrophobic chains (7-18 atoms of carbon), giving them an amphiphilic character and polymorphic properties. Evaluation of their physicochemical properties such as solvo-surfactivity and their ability to give to surfaces a textured morphology give them a superhydrophilic character.

\section{Lipochemical networks of synthesis of c-3 agrosynthons from glycerol}

The transformation pathways of natural glycerin for the directed synthesis of agro-C3 highly reactive synthons are illustrated in figure 1 (Mouloungui, 2004; Vriet and Mouloungui, 2008). The both major synthons, listed as top value added chemicals by the US Department of Energy (Werpy and Petersen, 2004) are glycerol carbonate and glycidol. The study of their reactivity opens the door to the synthesis and development of non-ionic interfacial agents that are esters of glycerol and fatty acids, pure monoglycerides, and esters of glycerol carbonate and fatty acids. The development of this C-3 glycerol based chemistry by the Mouloungui's group since 1994 (Mouloungui, 2004) was in respect of twelve principles of the green chemistry (Anastas and Warner, 1998), with atom economy, synthesis without solvent, and $\mathrm{CO}_{2}$ sequestration by organic carbonate production. These studies are referenced and described in a recent review (Corma et al., 2007).

\section{Catalytic synthesis of glycerol carbonate by transcarbonatation of glycerol or carbonylation of glycerol}

Glycerol carbonate is the first step in the chain generation of glyceryl synthons. Reactive glycerol carbonate is obtained by the heterocyclization of two vicinal hydroxyl groups of glycerol in cyclocarbonates by reacting glycerol with sources of organic or inorganic carbon-

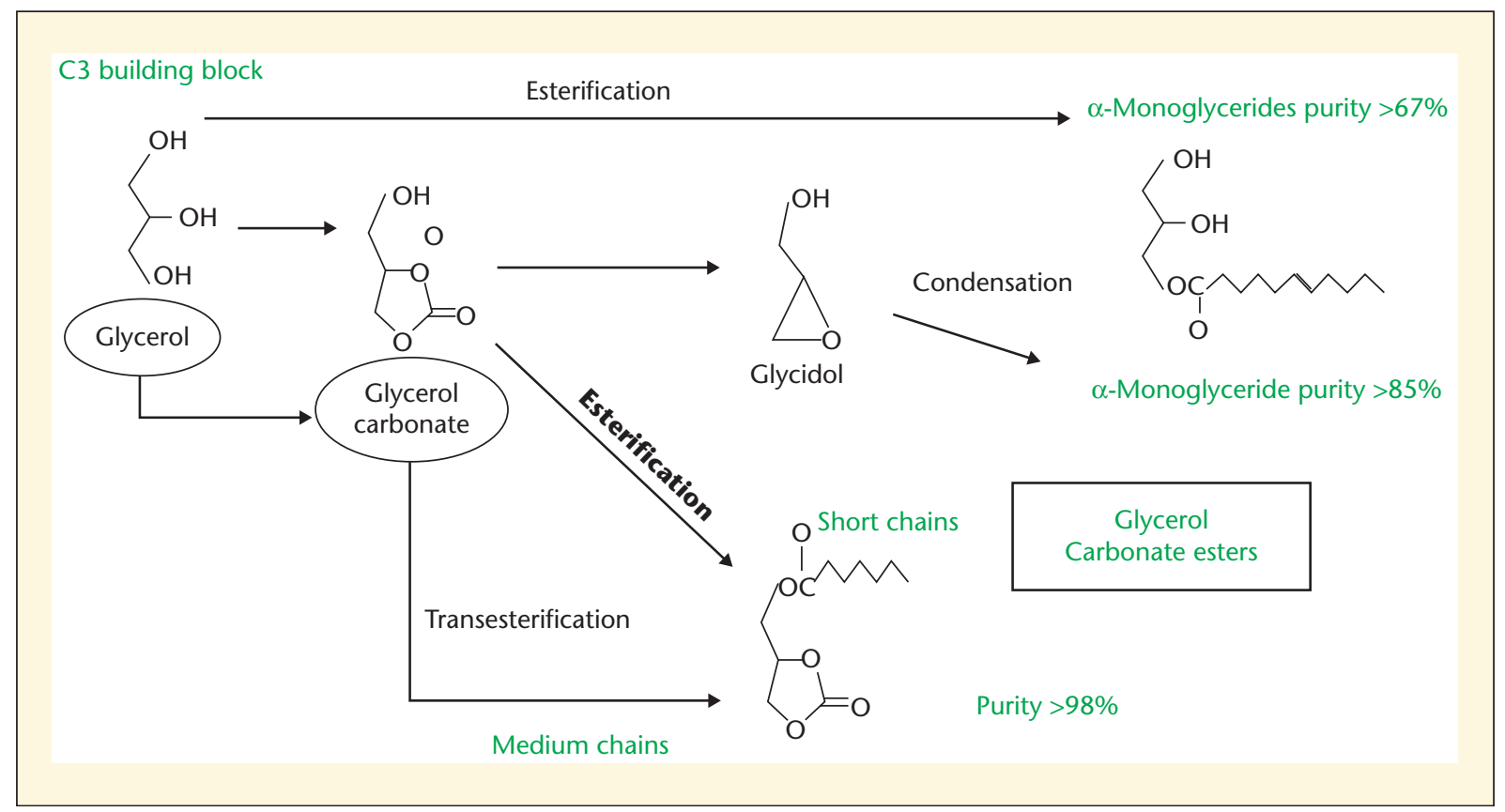

Figure 1. Transformation pathways of natural glycerin for the directed synthesis of agro-C3 highly reactive synthons. 
ate. Depending on the nature of carbonate donor, there are two methods of heterocyclization of glycerol: catalytic transcarbonation of glycerol by organic carbonates (Mouloungui et al., 1996; Vieville et al., 1998) and catalytic carbonylation of glycerol by urea (Claude et al., 2001, Mouloungui and Yoo, 2007)). Carbonation of glycerol by urea catalyzed by zinc sulfate is part of a systematic approach to improving the competitiveness of the method of synthesis of glycerol carbonate. Urea is a co-reactive, mineral carbonate donor and inexpensive. It is the masked form of $\mathrm{CO}_{2}$. Thus, the "urea method" contributes to the aliphatic sequestration of carbon dioxide in glycerol carbonate. The study and understanding of the mechanism of the reaction of urea with glycerol have improved the yield of the reaction and efficiency of the process. The catalytic process is based on the use of zinc sulfate and knowledge of the nature, number and strength of the reusable Lewis catalytic sites. The use of metal sulfates provides heterogeneous catalytic systems. Catalytic carbonylation of glycerol occurs in two consecutive steps: in situ carbamoylation and carbonylation of glycerol (Yoo and Mouloungui, 2003). The kinetics of formation of the glycerol carbonate and gaseous ammonia are comparable. Nucleation of zinc sulfate and the production of gaseous ammonia result in a change of the composition of the reaction system. The production of glycerol carbonate is increased by shifting the equilibrium of the reaction modulating the pressure at constant temperature.

\section{Catalytic synthesis of glycidol by oligomerization of glycerol carbonate}

Produced industrially by laborious processes of synthesis and purification from allyl alcohol of petrochemical origin, glycidol is now the second generation synthon in our integrated scheme. Work on the oligomerization of glycerol carbonate followed by the depolymerization thanks to the catalytic action and confinement effect of zeolites have open the way for the development of a new method for the synthesis of glycidol. The one-pot synthesis of glycidol (Yoo and Mouloungui, 2001) is carried out in liquid multiphase heterogeneous catalysis (glycerol carbonate/ glycerol $85 / 15 \mathrm{w} / \mathrm{w}$ )/solid (zeolite A)/gas ( $\mathrm{CO}_{2}$-glycidol) according the following five steps: i) diffusion of glycerol carbonate and glycerol to the catalytic sites of the zeolite A, ii) adsorption of molecules at the surface of the zeolite A, iii) surface reaction between glycerol carbonate and glycerol iv) desorption and diffusion of glycidol and gaseous carbon dioxide gas, and $v$ ) recycling of glycerol and sites of the zeolite A. A degree of glycidol 99\% was isolated after flash purification (Yoo and Mouloungui, 1998).

The both methods manufacture unit of glycerol carbonate and glycidol from glycerol are economical, clean and productive. They contribute significantly to control the price of the biodiesel glycerin and lower prices of these two building blocks. The availability of glycerol carbonate and glycidol allow the study of their reactivity and transformation into more elaborate molecules like monoglycerides and glycerol carbonate esters.

\section{Catalytic synthesis of pure monoglyceride by condensation of glycidol with fatty acids}

In view of their polyfunctional nature and their emulsifying, complexing, and lubricating properties, fatty acid monoglycerides find application in the food (Hartunian-Sowa et al., 1990; Fujimura et al., 1991), cosmetics, pharmaceutical (Rieger, 1990; Kabara and Lie, 1977; Kato, 1981) and textile and fiber industries (Kamei, 1990; Mc Intire, 1989). In certain applications, their efficacy hinges on the incorporation of pure monoglycerides (>90\%) as additives (Mouloungui and Gauvrit, 1998). All of the conventional methods for the preparation of monoglycerides, either by direct esterification of glycerol by fatty acids or by hydrolysis or transesterification of oils, lead to mixture of glycerides. Pure monoglycerides are generally obtained by molecular distillation, which raises their cost. An alternative chemical method is to prepare the monoglycerides from glycidol. In general, this reaction is conducted in the presence of a basic catalyst in a homogeneous medium: amines and/or quaternary ammonium salts (Lok, 1980), metal alcoholates (Burgos et al., 1987; Caron and Sharpless, 1985), or bases (Tamura, 1992). Although the yields of the glycerol monoesters are relatively satisfactory, the operating conditions in the homogeneous phase require phase-transfer agents and solvents that are able to withstand high temperatures. In heterogeneous catalysis, amines supported on mesoporous materials present good results, especially with hydrophobic surfaces (Cauvel et al., 1997). We develop the synthesis of pure monoglycerides, by direct condensation of fatty acid, like oleic acid described in the figure 2 with glycidol in aqueous medium in the presence of an anionexchange resin as a recyclable catalyst (Mouloungui et al., 2009). The new objective in organic chemistry is the development of reactions using water as the solvent instead of organic liquids. Of course, glycidol is a solvent of oleic acid, and glycidol and water are also miscible. Glycerol monooleate (GM-C18:1) and water produce emulsions. The condensation of oleic acid with glycidol to form GM-C18:1 is heterogeneously catalyzed by anion-exchange resins. The following processes are involved in the overall reaction: (a) diffusion of reagents to the catalytic sites of resins, (b) adsorption of reagents in the catalytic sites of the resin, (c) reaction at the surface between adsorbed reagents, (d) diffusion of the reaction products from the pores to the external layer of the resin, and (e) desorption of the reaction products into the liquid phase. The reaction on the surface (process $c$ ) is assumed to be the sole stage determining the kinetics. Hydrophobic-hydrophilic interactions of the molecules of the organic reactants were involved in the pseudoternary aquiorganic system. Two types of adsorption phenomenon can explain the interactions between glycerol monooleate and resin on one hand and oleic acid and resin on the other hand: physisorption of glycerol monooleate in the outer layer of resin and chemisorption of oleic acid. The marked swelling of the anionic resin with oleic acid indicates that the fatty acid penetrated the resin and that oleic acid was in contact with the active sites, where it was adsorbed and activated. We also noted a marked elimination of water during the phase of preadsorption of oleic acid onto the Ambersep 900-X $\left(\mathrm{X}=\mathrm{OH}^{-}, \mathrm{Cl}^{-}, \mathrm{HCO}_{3}^{-}\right.$) anion-exchange resins. Oleic acid thus appears to effectively desiccate the polymeric lattice, thereby enhancing the organohydrophobic nature of the solid polymeric catalyst. Then, adsorption of the fatty 


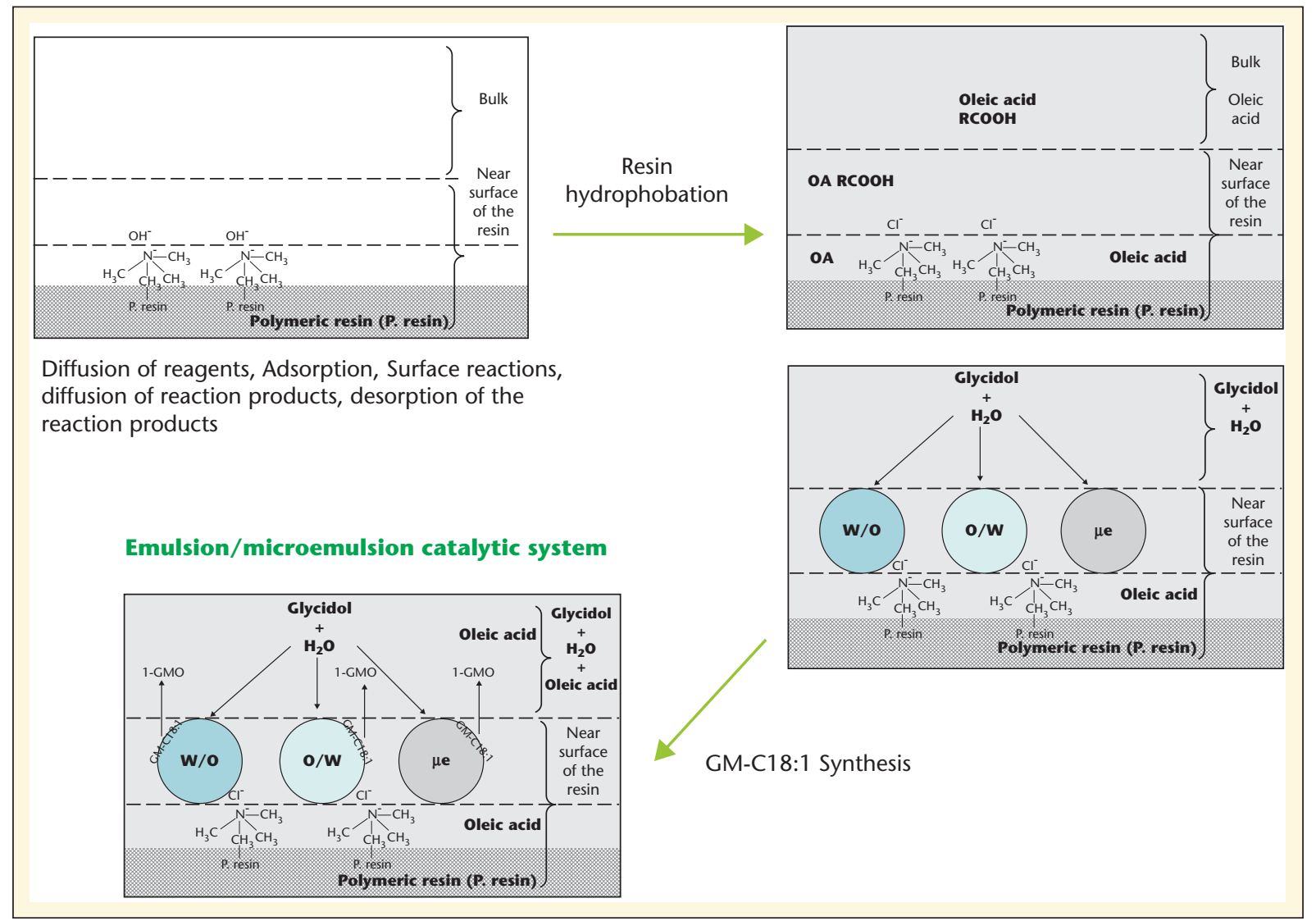

Figure 2. Physicochemical evolution of the condensation reaction of glycidol with oleic acid in the presence of Ambersep 900-Cl resin, (w/o, water/oil; o/w, oil/water; $\mu$ e, microemulsion). Resin before reaction (hydrophilic resin). Adsorption of oleic acid on resin (hydrophobic resin). Contact of glycidol/water solution with resin (hydrophilic/hydrophobic resin). Resin surrounded by polymorphic system (w/o system, o/w system, or microemulsion system) (Mouloungui et al., 2009).

acid is governed by van der Waals-type forces between the fatty hydrophobic hydrocarbon chains of the acid and the cross-linked polystyrene matrix, giving rise to a strong adsorbing capacity. The long-term stability of the resins is thus increased, with an enhanced catalytic system. By preadsorbing oleic acid onto the resin, the stage of transport and diffusion (stage a) of the fatty acid is no longer a limiting step in the overall reaction. The ensuing condensation between oleic acid and glycidol is a surface reaction. It takes place between reactants within the droplets dispersed in the continuous organic hydrophobic pseudophase within and on the surface of the macroporous particles and gels. The kinetics appeared to obey those described by the Langmuir-Rideal model for the surface reaction. The conditions were optimized for a discontinuous process in a stirred reactor. The highest yield of GM-C18:1 (97\%) was obtained with the non-functionalized form Ambersep $900-\mathrm{Cl}^{-}$at $70^{\circ} \mathrm{C}$.

\section{Catalytic synthesis of pure} monoglycerides by esterification of glycerol with fatty acids

An improved method for the production of monoglycerides by direct esterification of fatty acids with glycerol was developed (Eychenne and Mouloungui, 1999). The reaction medium was composed of oleic acid ( $72 \%$ purity), glycerol, dodecylbenzene sulfonic acid (DBSA) as catalyst and emulsifying agent, as well as of a molecular sieve as drying agent. Partial esterification of glycerol by oleic acid is carried out in either a batch reactor and in a column filled with a molecular sieve according to a continuous process. In a discontinuous batch reactor, system gives $28 \%$ of glycerol monooleate and strong proportions of di- and triesters. The addition of the $3 \AA$ molecular sieve to this system makes it possible to increase the selectivity in glycerol monooleate (47\%). However, the reaction carried out in a column filled with a $3 \AA$ molecular sieve produced a mixture containing $60 \%$ of glycerol monooleate after deacidification and removal of the catalyst and shows the advantage of a continuous process.

\section{Synthesis of glycerol carbonate} fatty acid esters by acyl transfert reaction of fatty acids or fatty esters with glycerol carbonate

Esters of glycerol carbonate have been relatively little reported. The long-chain carbonate esters are obtained by reaction of glycerol carbonate with acid chlorides (Oehlenschläger and Gercken, 1979; Mouloungui and Pelet, 2001). Other synthesis routes have been described: acylation of glycerol carbonate by acetic anhydrid (Gahe and Lachowicz, 1989), esterification by carboxylic acids (Lachowicz and Gahe, 1991), transesterification by methyl esters (O'Brien and Beavers, 1961). We emphasize that glycerol carbonate form with acids and fatty esters immiscible 
binary systems (Silvestre et al., 2010). Different parameters were studied: i) the solubility of these reagents by the determination of solubility parameters and the radius of the Hansen solubility sphere of glycerol carbonate, fatty acids and methyl oleate oleic sunflower, ii) the compatibilizing power of the glycerol carbonate ester (final product) on the coreactive evaluated by the study of the evolution of the interfacial tension of systems like glycerol carbonate/fatty acid/glycerol carbonate ester or glycerol carbonate/fatty ester/glycerol carbonate ester, depending of the studied reactions: esterification and alcoholysis (Mouloungui and Pelet, 2001). The results explained that glycerol carbonate is immiscible with, reactants such as fatty acids and fatty esters, and the producted glycerol carbonate ester. The glycerol carbonate is a mono-alcohol with high polar properties. It is a donor-acceptor of hydrogen bonds. To allow the reaction to take place, it is important to involve amphiphilic catalysts, penetrating the pseudo-ternary mixtures of glycerol carbonate/fatty acid-glycerol carbonate ester or glycerol carbonate/fatty estersglycerol carbonate ester. The para-toluene sulfonic acid was found to be an amphiphilic catalytic agent with the ability to organize the glycerol carbonate medium in the octanoic acid. This lead to a hydrophilic zone embedded in a hydrophobic medium constituted of the amphiphilic glycerol carbonate octanoate. The esterification reaction takes place simultaneously at the interface of glycerol carbonate/octanoic acid and in the hydrophobic phase fatty acidglycerol carbonate ester. Using amphiphilic organotin catalysts such as dibutyltin oxide, the acylation of glycerol carbonate by alcoholysis also involves the solubilization of glycerol carbonate in the hydrophobic phase: glycerol carbonate methyl ester-glycerol carbonate oleate organized in reverse micelles. The quantitative synthesis of glycerol carbonate esters with yields of $60-95 \%$ is interpreted in this way through the establishment of non-covalent bonds.

\section{Physico-chemical properties}

\section{Molecular conformation}

Monoglycerides and glycerol carbonate esters are two families of bifunctionnal

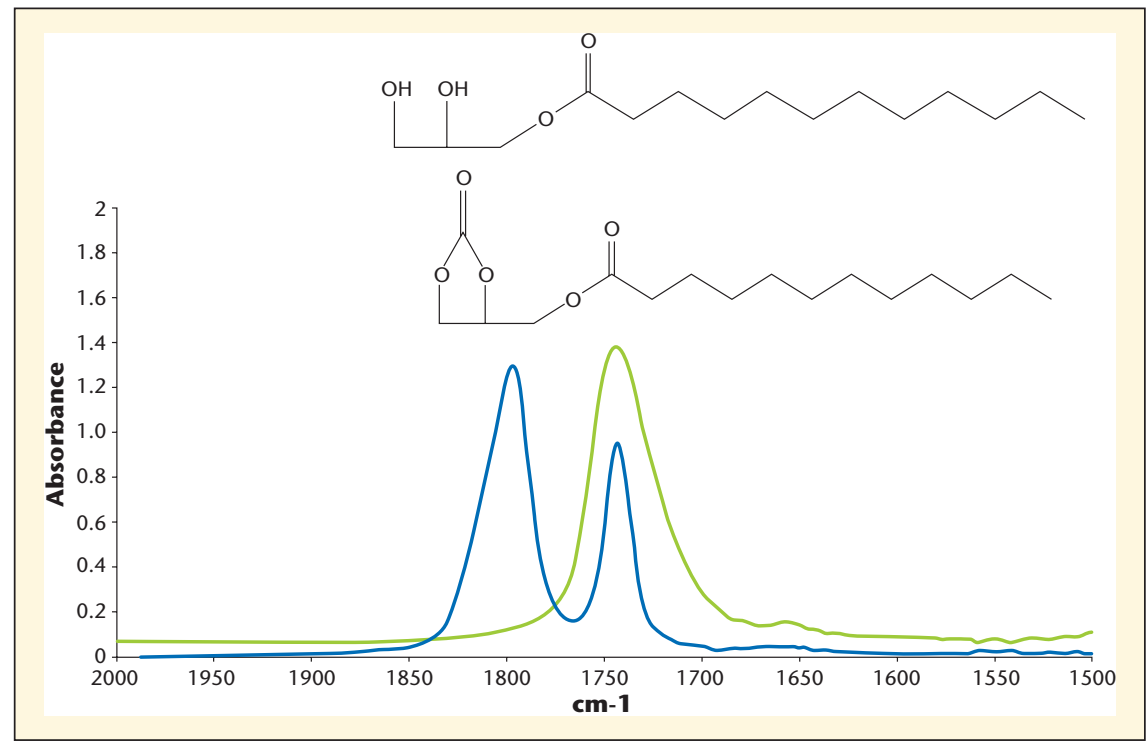

Figure 3. Transmission FTIR analysis of glycerol monolaurate and glycerol carbonate laurate in DMSO. Concentration in fatty acid ester $\approx 0.01 \mathrm{mg}^{\mathrm{mL}} \mathrm{L}^{-1}$. Bruker Tensor 27 apparatus equipped with DTCS detector was used.

biomolecules. The hydrophobic part of monoglycerides is a hydrocarbon chain and the hydrophilic component is a glycerol unit. The hydrocarbon chain is provided by the plant fatty acids. They are inserted in position 1 of the glycerol. The glyceryl polar head containing two free hydroxyl and the lipophilic fatty acid chain give to monoglycerides an amphiphilic character. Glycerol carbonate esters differ because they are monoglycerides of fatty acid esters in which the hydroxyl functions are blocked. They are oleophilic compounds in which the hydrophobic moiety is a hydrocarbon chain and the hydrophilic part is the glycerol cyclocarbonate head. Chemically, monoglycerides consist of an exocyclic ester linkage between the hydrophobic part and the polar head. Glycerol carbonate fatty acid esters consist of an exoxyclic ester linkage between the hydrophobic part and the polar head containing two endocyclic ester functions. These both type of ester functions can be discriminated by FTIR spectroscopy (figure 3). The endocyclic esters (Oki and Nakanishi, 1971), are characterized by a band at $1797 \mathrm{~cm}^{-1}$ whereas the exocyclic ester, give à vibrational band at $1743 \mathrm{~cm}^{-1}$. The conformation variability of the polar heads, glycerol or glycerol carbonate, were very interesting because this impacts the hydrogen bonding capacity. At interfaces and in presence of water, physico-chemical properties could be affected.

\section{Polymorphism of pure molecules}

There is no long-chain compound which is not polymorphic, and this property is particularly pronounced in fats and lipids. The crystallization behavior of fatty molecules (the crystallization rate, crystal size and network, crystal morphology and crystallinity) is directly influenced by the polymorphism; polymorphism depends on the molecular structure itself and on several external factors such as temperature, pressure, solvent, the rate of crystallization, and impurities. Differential scanning calorimetry (DSC) is a technic which allows highlighting the different polymorphisms. A DSC Mettler Toledo DSC1Star e System apparatus (Perkin Elmer, USA) equipped with an IntraCooler cooling system was used. The purge gas was nitrogen at a rate of 20 $\mathrm{ml} / \mathrm{min}$. Indium $\left(\mathrm{Tm}=156.6^{\circ} \mathrm{C}, \Delta H_{f}=\right.$ $28.45 \mathrm{~J} / \mathrm{g})$ and distilled water $\left(\mathrm{Tm}=0^{\circ} \mathrm{C}\right.$, $\Delta H_{f}=333.79 \mathrm{~J} / \mathrm{g}$ ) were used for calibration. Data were analyzed using Pyris software (Perkin Elmer). Samples of $c a$. 4-10 mg were weighed into aluminum pans to the nearest $0.1 \mathrm{mg}$, and covers were hermetically sealed into placed with O-rings. An empty, hermetically sealed aluminium pan was used as the reference. To erase the thermal history 
of the samples, they were heated to $80^{\circ} \mathrm{C}$ for $5 \mathrm{~min}$ before the analysis of thermal behavior. Successive cycle of cooling and heating were applied with different cooling and heating rate: $1^{\circ} \mathrm{C} / \mathrm{min}, 5^{\circ} \mathrm{C} / \mathrm{min}, 10^{\circ} \mathrm{C} / \mathrm{min}$ and $20^{\circ} \mathrm{C} / \mathrm{min}$.

Monoglyceride polymorphism is well known (Malkin, 1954). As show in figure 4 , the glycerol monolaurate present four polymorphs, sub $\alpha, \alpha, \beta^{\prime}$, $\beta$. The $\beta$ form corresponds to the more stable form and was attributed to the melting point of the molecule. At low cooling and heating rate $\left(1^{\circ} \mathrm{C} / \mathrm{min}\right)$, the more stable form $\beta$ appears preponderant. Increasing the cooling and heating rate, $1^{\circ} \mathrm{C} / \mathrm{min}$ to $20^{\circ} \mathrm{C} / \mathrm{min}$, other less stable forms are present. They are noted in order of stability sub $\alpha, \alpha, \beta^{\prime}$ and $\beta$. Monoglycerides differ from triacylglycerol which present generally three polymorphs. We compared the thermal behavior of pure monoglycerides (figure 4) to that of pure glycerol carbonate esters (figure 5). As show for the glycerol carbonate laurate, glycerol carbonate fatty acid esters present four polymorphs, and it is clearly visible in the figure 5 that the height of the peak corresponding to the more stable form (melting temperature) is very more intense than other transitions whatever be the cooling and heating rates. This behavior differs from that of monoglycerides. It appears that glycerol carbonate esters are few sensible to thermal events. This is the result of the blocking of the hydroxyl function on the polar head of glycerol carbonate fatty acid ester. Same experiments were performed on GCE-Cs of lower chain length C8, C9 and C10 and we noted on figure 6 the temperature of the thermal transitions.

All thermograms present very similar shapes with same number of phase transitions. For each glycerol carbonate ester, four temperature transitions were found. All increased with the chain length. The more intense form 4 corresponding to the melting transition is always preponderant whatever may be the scanning temperature rates. The melting temperature increased with the chain length, nor linearly (figure 6). Melting temperature values of GM-Cs were lower than the melting points of same chain length monoglycerides.

We studied in more detail thermal behavior of glycerol carbonate laurate

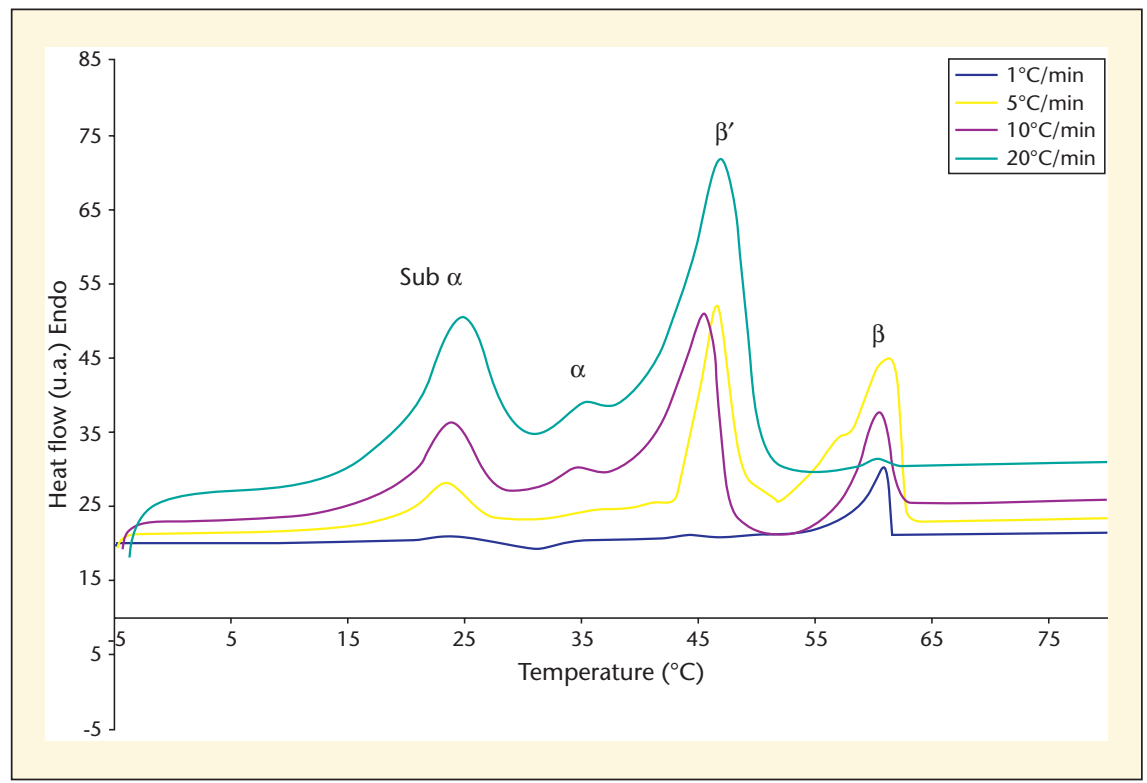

Figure 4. Thermogramms of glycerol monolaurate (GM-C12). with Polarized light microscopy. A Nikon Eclipse E600 optical microscope (Nikon Corporation, Japan) was used equipped with polarized filters and a Mettler Toledo FP82HT hot stage managed by a FP90 central unit. The images (figure 7) were acquired with a high resolution, low noise CCD Nikon DXM1200 color camera and analyzed using LUCIA G version 4.8 software. The central processor unit was programmed to heat the sample at a heating rate of $5 \%$ min until the melting of the sample. Images were acquired with a magnification of hundred times at each $1^{\circ} \mathrm{C}$.
Temperature scans of samples were carried out with polarized light microscopy aiming at identifying the phase transitions observed using DSC. Below $49^{\circ} \mathrm{C}$, all images show the sample completely solid, being possible to observe the crystals in thin overlapped layers and presenting an irregular shape. At $25^{\circ} \mathrm{C}$, ambient temperature, the crystal was observed after the first transition (form 1 in DSC). At $30^{\circ} \mathrm{C}$, morphology of crystals changes and the image became brighter. Another phase transition was observed at $37^{\circ} \mathrm{C}$ according the DSC data (form 3 ) and

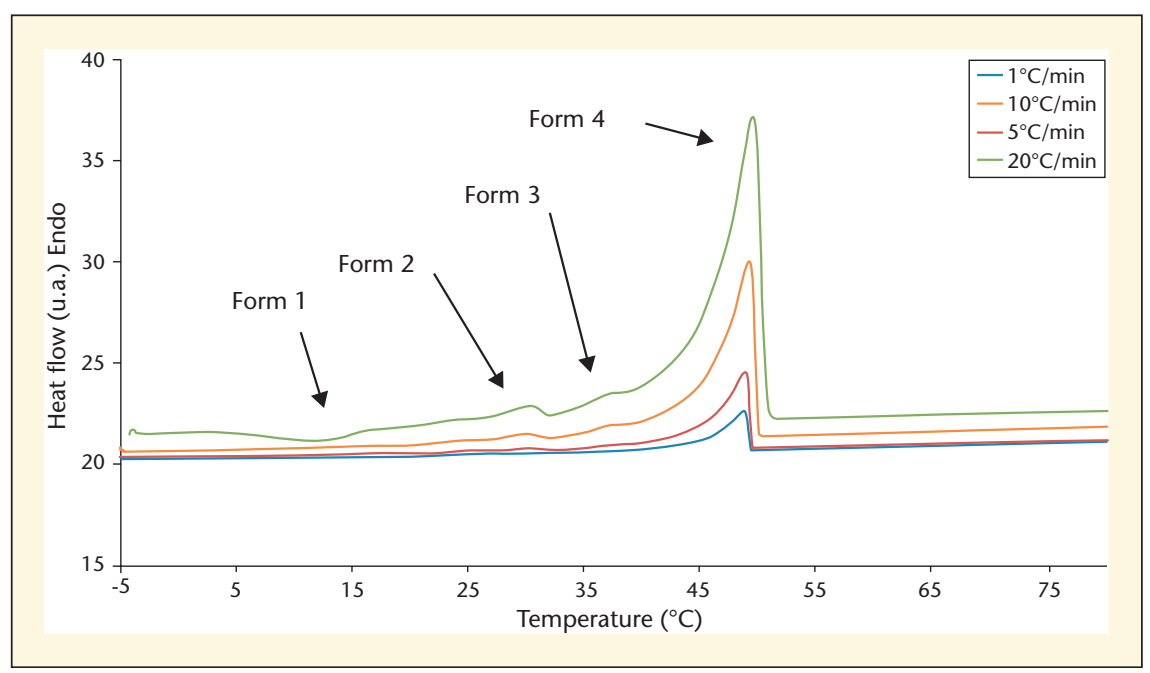

Figure 5. Thermogramms of glycerol carbonate laurate (GCE-C12). 


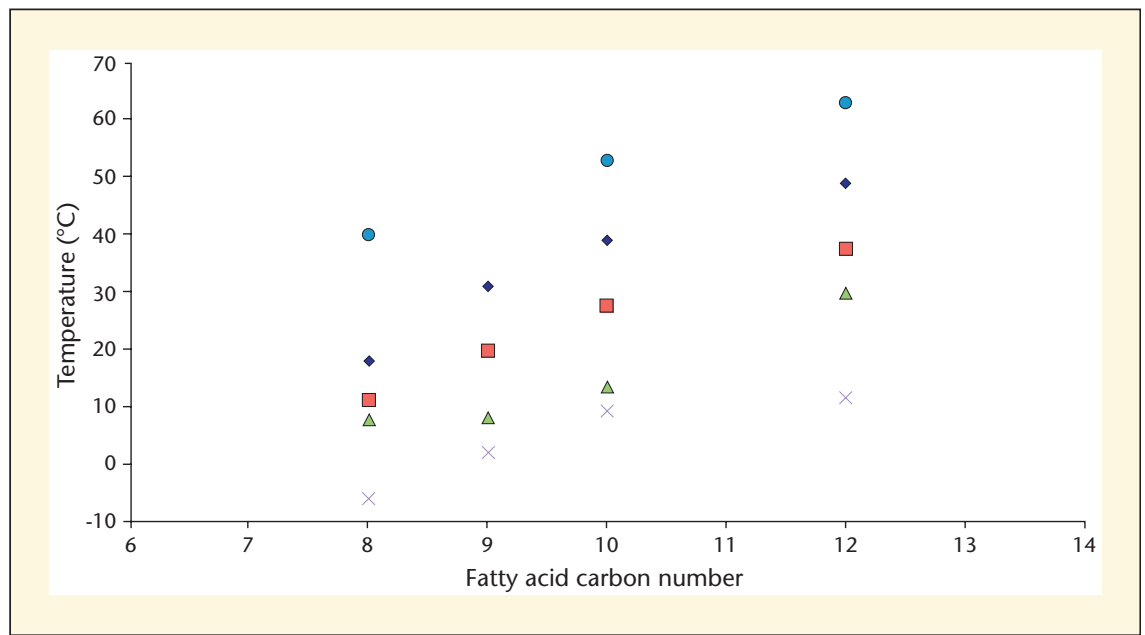

Figure 6. Transition temperatures ( $\times$ form 1, $\triangle$ form 2, $\square$ form 3 and $\diamond$ form 4 ) observed by DSC for fatty acid glycerol carbonates esters of different chain length GCE-C8, GCE-C9, GCEC10, GCE-C12 and X melting point temperature of monoglycerides.

finally at $49^{\circ} \mathrm{C}$, the last phase transition before melting was observed. Here, it seems that numerous crystals present similar morphology. It is noting that during heating, no liquid phase appeared which means that phase changes were solid/solid. Probably glycerol carbonate laurate crystals are composed of a mixture of phases with different crystallographic characteristics. To identify them, a study by $X$-Ray diffraction should be carried out.

\section{Self-assembling with water}

When heating monoglycerides and glycerol carbonate esters in presence of water above their melting point or Kraft point, coagel can be obtained. Coagels are made up of densely packed lamellar layers of surfactants interlocked by hydrophilic regions that contain a specific amount of strongly bound water molecules. The stability of monoglyceride layers is related to the specific amount of the "frozen" water molecules. DSC experiments provide informations on the number of hydration water molecules strongly interacting with the amphiphilic headgroups through hydrogen bonding (Ambrosi et al., 2004). In formulation the crystals are linked in connective domains or junction zones, probably containing all of the material. Each substance acts either by substituting part of the hydration water molecules, or by insertion within the hydrophobic tail domain or in the hydrophilic region. This stabilizes the hydrated mixture of fatty acid esters on the surfaces. Calorimetric studies showed the presence of two types of water in the coagel phase: bulk or free water that freezes and then melts at about $0{ }^{\circ} \mathrm{C}$, and frozen, strongly bound water that does no melt in the temperature range investigated. DSC experiments allow the determination of the percentage of strongly bound water per hundred grams of sample, $W_{b}(\%)$. It can be calculated from the decrease of the area of the endothermic peak associated with the melting of bulk water, as follows:

$$
W_{b}(\%)=\frac{333.79-\Delta H_{\exp }}{333.79}(100-P)
$$

Here $333.79 \mathrm{~J} \mathrm{~g}^{-1}$ is the heat of melting of pure water (Dean, 1985) and $\Delta \mathrm{H}_{\mathrm{exp}}$ is obtained from the measured peak area for each coagel at a glycerol carbonate ester concentration $\mathrm{P}$. The number of strongly bound water molecules per surfactant polar headgroup, $\mathrm{N}$, can be calculated as follows: $N=\left(M_{n} W_{b}\right) /\left(M_{w}\right.$ P) where $M_{w}$ and $M_{n}$ are the molecular weights of water and glycerol carbonate ester, respectively. Strongly bound water molecules form a thin compartment sandwiched between the surfactant bilayers, whereas bulk water surrounds the plate like "islands" and dissolves the few monomers in equilibrium with the coagel phase. The gelation of monoglycerides has been studied (Krog, 1975), and it can be calculate from the determined diffraction parameters that 14.4 molecules of water per molecule of monoglyceride are involved in $60 / 40 \mathrm{w} / \mathrm{w}$ monoglyceride-water. In the same conditions replacing glycerol monolaurate by glycerol carbonate laurate, this value decrease to 4.4 molecules of water per molecule of glycerol carbonate laurate as shown in figure 8 .

These results are consistent with the chemical nature of monoglycerides and glycerol carbonate esters. Monoglycerides are donor-acceptor of hydrogen bond whereas glycerol carbonate esters are only acceptor. Monoglycerides have the ability to "freeze" more water molecules than glycerol carbon-

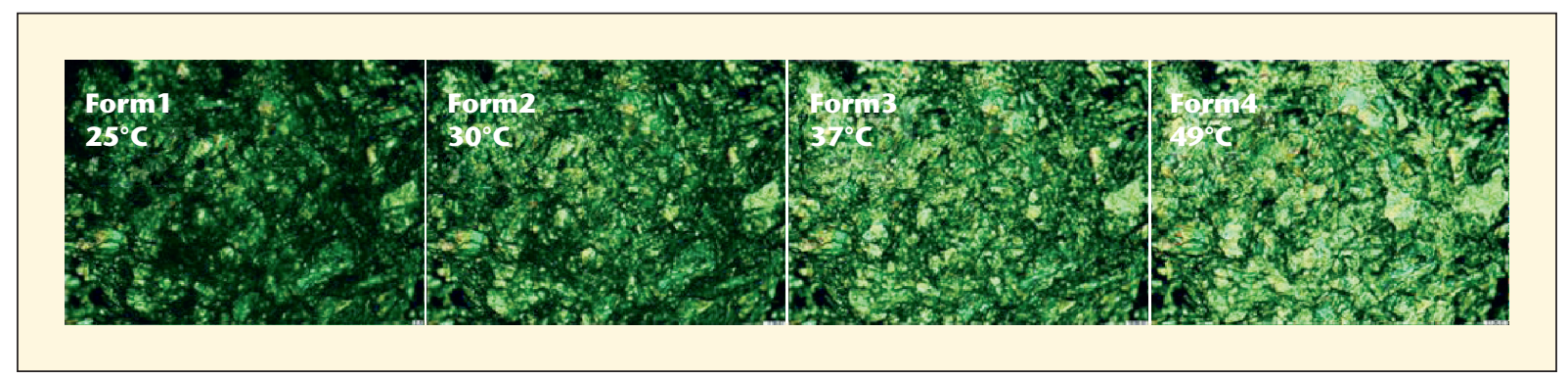

Figure 7. Light microscopy images of glycerol carbonate laurate. Magnification 100 times. 


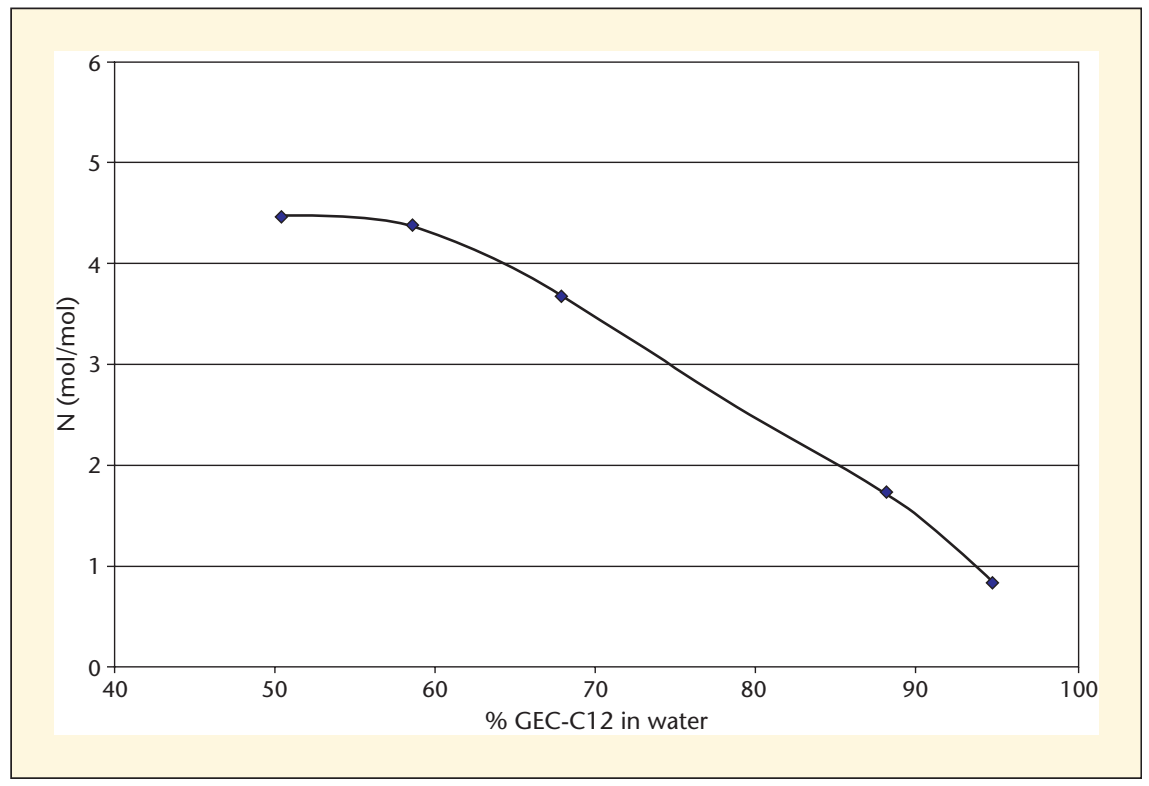

Figure 8. Number of strongly bound water molecules per molecule of glycerol carbonate laurate. ate esters. By self-aggregation, glycerol carbonate laurate is an efficient waterreppellent molecule.

\section{Surface activity}

Surface tension measurements allow the determination of several important parameters. Accepting that Gibbs adsorption model describes the behavior of micellization for esters, from the plot of surface tension values against the natural logarithm of concentration

(figure 9), before micellization, it is possible to determine the surface excess and the molecular area, according equations:

$$
\Gamma=-\left(\frac{1}{R T}\right)\left(\frac{\partial \gamma}{\partial i n C}\right)_{T, P} \text { and } A=\frac{1}{N_{A} \Gamma}
$$

Where $\Gamma$ is the surface excess, $A$ the molecular cross-sectional area, $\mathrm{N}_{\mathrm{A}}$ the Avogadro's number, $T$ the temperature, $\mathrm{R}$ the gas constant, $\mathrm{P}$ the pressure and $\mathrm{C}$ the concentration.

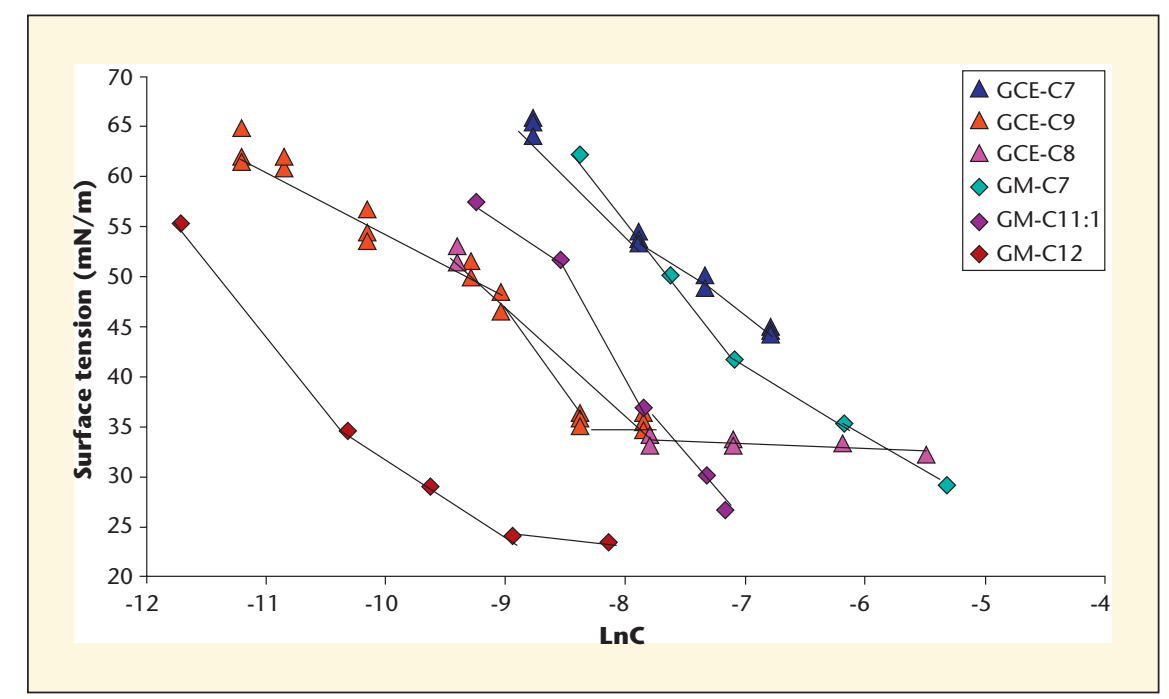

Figure 9. Surface tension (by Wilhemy plate technique) vs natural logarithm of the concentration $\mathrm{C}$ (in $\mathrm{mg}^{\mathrm{mL}} \mathrm{L}^{-1}$ ) at $25^{\circ} \mathrm{C}$.
Figure 9 shows profiles obtained for surface tension variations upon monoglycerides and glycerol carbonate esters. Surface excess values were determined where a linear dependence between surface tension and concentration natural logarithm was observed. It has to be stressed that the mechanism of aggregation (e.g., micellization, pseudophase separation, etc.) does not affect the process of estimating $A$ since all the data points that are taken for the calculations correspond to concentrations lower than the concentration at which the aggregation process starts. For the solutions that remained clear, the critical aggregation concentration (CAC) is expected to correspond to the critical micelle concentration (CMC). In the case of solutions that appeared turbid in the vicinity of the concentration where a surface tension plateau was observed, the term CAC can still be applied because a reversible aggregation process was taking place and the surfactants can also called solvo-surfactants (Queste et al., 2006). For reasons of consistency we will be describing all of the compounds studied in terms of their CAC and the surface tension at the CAC. The CAC in this study has been graphically determined by plotting surface tension against the logarithm of the weight/volume concentration. Two straight lines were fitted to the steep and the (nearly) flat part of the surface-tension curves. The results are presented in table 1. The data plotted in figure 9 can be used to produce estimates of the cross-sectional area of the surfactant molecules at the interface, assuming their adsorption is described by the Gibbs adsorption isotherm. These values are shown in table 1. Glycerol carbonate esters have the bulkiest headgroup at the interface. The calculated value of $\mathrm{A}$ for glycerol heptanoate (GM-C7) is $25.6 \AA^{2}$ lower of value obtained for glycerol carbonate heptanoate $38.7 \AA^{2}$. Knowing $A$, it is possible to predict the geometry of the aggregates by applying the critical packing parameter criterion, CPP (Israelachvili et al., 1976).

CPP is calculated by the:

$$
C P P=\frac{v}{\mathrm{~A} / \mathrm{c}}
$$

where $v$ is the volume of the hydrocarbon chain, $A$ is headgroup area, and $I_{c}$ 
Table 1. Physicochemical properties of monoglycerides and glycerol carbonate esters $\left(25^{\circ} \mathrm{C}\right)$.

\begin{tabular}{|c|c|c|c|c|c|c|c|c|}
\hline & $\begin{array}{l}\text { CMC/CAC } \\
(\mathrm{mmol} / \mathrm{L})\end{array}$ & $\begin{array}{l}\text { CMC/CAC } \\
(\mathrm{mg} / \mathrm{L})\end{array}$ & $\begin{array}{l}\text { A Area/molecule } \\
\left(\AA^{2}\right)\end{array}$ & $\begin{array}{l}\text { Vm } \\
\left(\AA^{3}\right)\end{array}$ & $\begin{array}{l}\mathbf{L} \\
(\mathbf{A})\end{array}$ & $\begin{array}{l}\gamma \mathbf{c m c} \\
(\mathbf{m N} / \mathbf{m})\end{array}$ & CPP & Geometry \\
\hline GM-C7 & 1.00 & 204 & 25.6 & 188.8 & 9.09 & 35.3 & 0.81 & Bilayers \\
\hline GM-C11:1 & 0.38 & 89.0 & 23.0 & 296.4 & 15.0 & 36.9 & 0.91 & Bilayers \\
\hline GM-C12 & 0.29 & 79.5 & 31.1 & 323.3 & 15.4 & 24.1 & 0.68 & Bilayers \\
\hline GCE-C7 & 1.13 & 259.9 & 38.7 & 188.8 & 9.1 & 44.4 & 0.54 & Bilayers \\
\hline GCE-C8 & 0.41 & 100.0 & 42.1 & 215.7 & 10.4 & 33.3 & 0.49 & Rods \\
\hline GCE-C9 & 0.25 & 64.5 & 60.0 & 242.6 & 11.6 & 35.5 & 0.35 & Rods \\
\hline${ }^{\mathrm{a}} \mathrm{C}_{9} \mathrm{COE}_{3}$ & 0.9 & 273.6 & 45 & - & - & 27.3 & 0.47 & Rods \\
\hline${ }^{\mathrm{a}} \mathrm{C}_{9} \mathrm{COE}_{4}$ & 0.8 & 278.4 & 50 & - & - & 28.5 & 0.42 & Rods \\
\hline
\end{tabular}

${ }^{a}$ (Zhu 2009).

is the average length of the hydrophobic tail. For hydrocarbon chains the following formulas can be used (Tanford, 1980),

$$
\begin{aligned}
& v=27.4+26.9 n(\text { in } \AA \text {, })\left(\text { in } \AA^{3}\right) \\
& I_{c} \leq I_{\max }=1.5+1.265 n(\text { in } \AA)
\end{aligned}
$$

Where $\mathrm{n}$ is the number of carbon atoms of the hydrophobic chain. The effective hydrophobic chain length of the fatty acids should be close to $n_{c}-1$, where $n_{c}$ is the number of carbon atoms in the fatty acid. The CPP predicts spheres, rods, bilayers, and inverse structures when CPP $<1 / 3,1 / 3<\mathrm{CPP}<1 / 2,1 / 2<\mathrm{CPP}<1$, and CPP $>1$, respectively. The results of the calculations are presented in table 1. It has to be stressed that the micelle shape derived from this method is just an estimate based on simple geometric considerations. The method provides no information on the polydispersity of the micelles present in the system.

Results showed that monoglycerides aggregate in bilayers. Glycerol carbonate esters, except CGE-C7 give aggregates in rods with CPP values close of esters of polyethylene glycol of same chain length $\left(\mathrm{C}_{9} \mathrm{COE}_{3}\right.$ and $\left.\mathrm{C}_{9} \mathrm{COE}_{4}\right)$ (Zhu, 2009). We noted too that glycerol carbonate esters polar head is a monomer unit "glycerol carbonate" while polyethylene glycol polar head is an oligomeric moiety of 3 or 4 ethylenic groups.

\section{Lipophilic activity}

A first estimation of the polarity of a substance can be performed by looking at its $\log P$ value. The octanol-water partition coefficient, $P$, expresses the differential solubility of a substance between these two immiscible solvents. It equals the ratio of the concentration of the substance solubilized in octanol over the concentration of the substance solubilized in water. Therefore, it is often used as a descriptor of the hydrophobicity. Monoglycerides can provide more links with water than glycerol carbonate esters as explained in previous part. This can be also illustrated in figure 10. LogP of monoglycerides and glycerol carbonate esters are compared. Both experimental and calculated values are plotted for GCE-Cs. Calculated values are lower than experimental values. GM-Cs $\log P$ values are furthermore lower than those of GCE-Cs. It can be deduced that GCE-Cs, for same atom carbon number on the fatty acid chain, are more lipophilic than GM-Cs.
We can suppose that monoglycerides are responsible of the stability of hydrated coagel in surface glycerol carbonate esters-monoglycerides formulations.

\section{Surface formulation}

Formulations of glycerol carbonate octanoate, with high affinity with polar surfaces and monoglycerides of high melting point like glycerol monolaurate, can be applied by simple deposition and confer to metal and polymer surface superhydrophilic properties (figure 11). We showed a synergy between melting and solvo-surfactive properties to obtain superhydrophilic surfaces (Valentin et al., 2012).

Water contact angle measurements (figure 11) showed that steel $(S)$ and

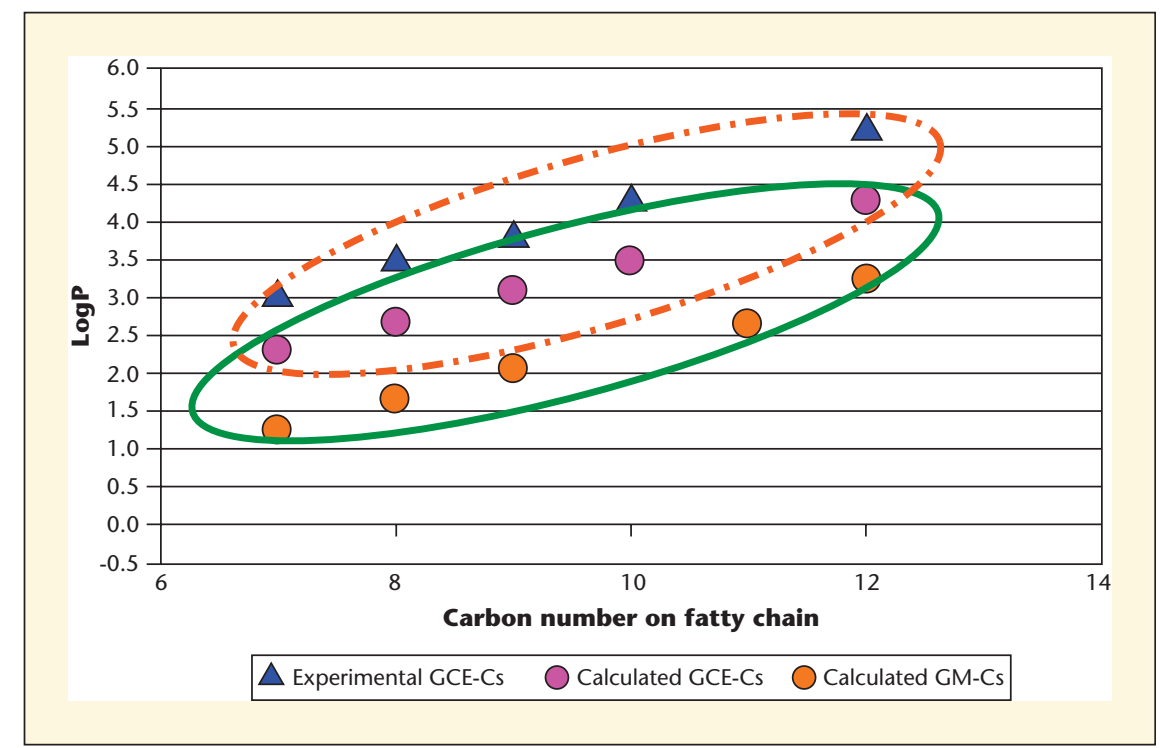

Figure 10. logP values of glycerol carbonate fatty acid esters and monoglycerides. Hyperchem ${ }^{T M}$ Release 7.01. HyperChem QSAR Properties v. 7.0 was used to calculate logP. Experimental values were carried up by liquid phase chromatography method on reverse C18 phase column. 


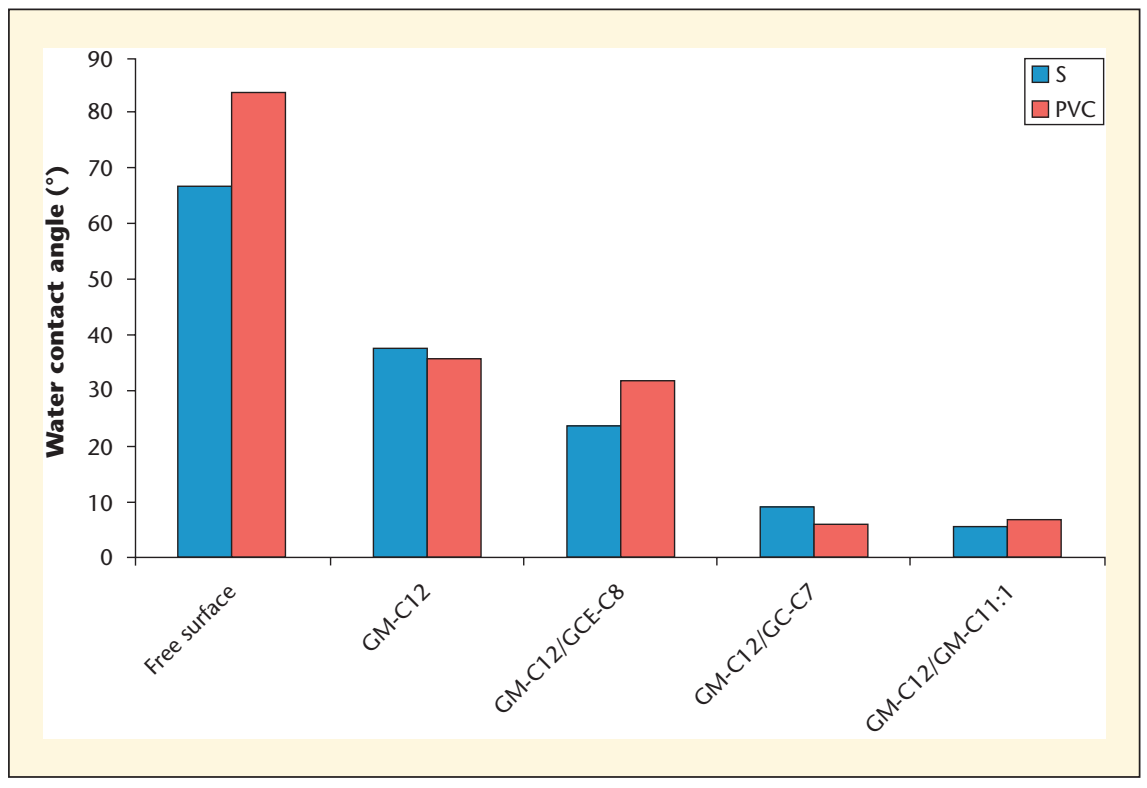

Figure 11. Water contact angles on steel (S) and polyvinyl chloride (PVC) surfaces.

polyvinyl chloride (PVC) surfaces, all coated with monoglycerides and formulations of monoglycerides and glycerol carbonate esters, exhibit lower contact angle values in comparison to uncoated surfaces. The quality of waterrepellent surfaces was improved by improving the purity of fatty acid esters. When using only high-purity grade fatty-acid esters, high leaching-resistant superhydrophilic surfaces could be obtained. The stability of the coating was due to the formation of stable coagels on the surfaces. This highly superhydrophilic coating was carried up by biomolecules containing odd numbers of carbon on their fatty acid chain GM-C7, GM-C11:1 and even numbers

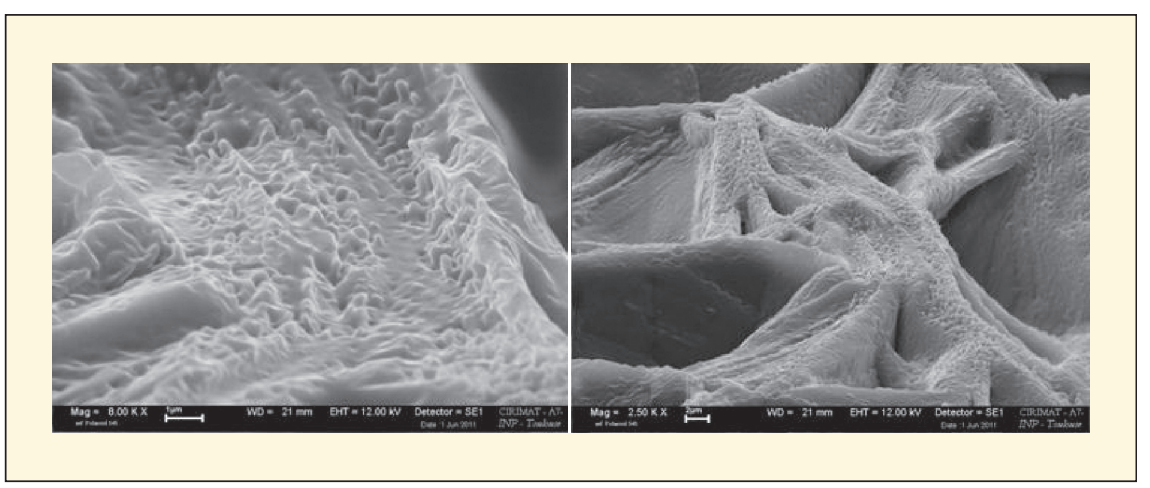

Figure 12. SEM images of glycerol monolaurate on steel surface. The sample is tilted at $70^{\circ}$ relative to the electron beam (Valentin et al., 2012). (figure 12). We can see numerous asperities of submicrometric dimension, range where capillarity phenomena taking place. Dong et al. used this concept by adding silica nanoparticles to relatively low hydrophilic polymers. Incorporation of the nanoparticles significantly decreased the contact angle due to the increase in surface roughness and resulting 3D capillary hydrophilicity (Dong et al., 2010).

Wettability by water is then enhanced which lead to increase the hydrophilicity of the surfaces. The concept of mixing capillarity phenomena with detergency can be considered as a biomimetic approach. Indeed, in Ruellia devosiana (Koch and Barthlott, 2009) the glands secreted surfactant substances that in combination with the surface roughness leaded to superhydrophilicity. The water droplet spreading coefficient $S$ was very close to 0 and in this case, a very low inclination of a few degrees of the covered surface was sufficient for a drop of water to flow spontaneously. On surfaces, in presence of water, multilayer deposited fatty acid esters form coagels (Sein et al., 2002; Ambrosi et al., 2004) and when a drop of water was deposited, the coagel behave like a water surface and the drop water meets this high hydrophilic coagel surface and spreads spontaneously.

\section{Conclusion}

The transformation pathways of natural glycerin for the directed synthesis of agro-C3 highly reactive synthons like glycerol carbonate and glycidol open the door to the synthesis and development of non-ionic interfacial agents that are esters of glycerol and fatty acids, pure monoglycerides, and esters of glycerol carbonate and fatty acids. The respect of the twelve principles of the green chemistry is achieved by the development of this C-3 glycerol based chemistry including atom economy, synthesis without solvent and use of the multifunctionality of the reactants and products. Indeed, solvo-thermal, hydroxy solvo-thermal and self-assembled reactive media can be use, without adding solvent and additional surfactants. Glycerol and glycerol carbonate are reactants which can act as solvents and thermal conductors. Monoglycerides and glycerol carbonate esters are reaction products and solvo-surfactant 
molecules that can play the role of solvent, surfactant and compatibilizing agent. We showed that glycerol carbonate esters are new polymorphic molecules different from monoglycerides. Carbonation decreases melting points: two hydroxyl functions are blocked on GCE-Cs while GM-Cs present two hydroxyl functions involved in intra and intermolecular hydrogen bonds. Carbonation decreases the hydrophilic character of the polar head in surface-active GCEs. Short chain and medium chain GM-Cs and GCE-Cs are solvo-surfactants molecules. They are durable water-repellent agents. Formulation of monoglycerides and glycerol carbonate esters on surfaces induce superhydrophilicity by biomimetism. This study open the way to new uses for theses self-assembled biomolecules like surface protection, stabilization by emulsification, transport of water and protic molecules.

\section{Disclosure}

Conflict of interest: none.

\section{REFERENCES}

Ambrosi M, Lo Nostro P, Fratoni L, et al. Water of hydration in coagels. Phys Chem Chem Phys 2004; 6: 1401-7.

Anastas PT, Warner JC. Green Chemistry: Theory and Practice. Oxford: Oxford University Press, 1998.

Bauer U, Bohn HF, Federle W. Harmless nectar source or deadly trap: Nepenthes pitchers are activated by rain, condensation and nectar. Proc $R$ Soc $B$ 2008; 275: 259-65.

Burgos CE, Ayer DE, Johnson RA. A new, asymmetric synthesis of lipids and phospholipids. J Org Chem 1987; 52: 4973-7.

Caron M, Sharpless KB. Titanium isopropoxide-mediated nucleophilic openings of 2,3-epoxy alcohols. A mild procedure for regioselective ring-opening. / Org Chem 1985; 50: 1557-60.

Cauvel A, Renard G, Brunel D. Monoglyceride Synthesis by Heterogeneous Catalysis Using MCM-41 Type Silicas Functionalized with Amino Groups. J Org Chem 1997; 62: 749-51.

Cebeci FC, Wu Z, Zhai L, Cohen RE, Rubner MF. Nanoporosity-Driven Superhydrophilicity: A Means to Create Multifunctional Antifogging Coatings. Langmuir 2006; 22: 2856-62.
Claude S, Mouloungui Z, Yoo JW, Gaset A. Process for the preparation of glycerol carbonate EP0955298(A1) 2001.

Corma A, Iborra S, Velty A. Chemical Routes for the transformation of biomass into Chemical. Chem Rev 2007; 107: 2411-502.

Dong H, Ye P, Zhong M, Pietrasik J, Drumright $\mathrm{R}$, Matyaszeski K. Superhrophilic surfaces via polymer- $\mathrm{SiO}_{2}$ nanocomposites. Langmuir 2010; 26: 15567-73.

Dean JA. Lange's Handbook of Chemistry. New York: McGraw-Hill Book Company, 1985.

Drelich J, Chibowski E. Superhydrophilic and superwetting surfaces: definition and mechanisms of control. Langmuir 2010; 26:186243.

Eychenne V, Mouloungui Z. High concentration of 1-(3-)monoglycerides by direct partial esterification of fatty acids with glycerol. Fett/Lipid 1999; 101: 424-7.

Fujimura M, Yamauchi H, Matsushita T, Oshima M, Oya K. Shortenings containing glycerides and amylase and/or protease and their manufacture. Japanese Patent JP 03292 848, 1991.

Gahe G, Lachowicz A. Esters containing cyclic carbonate, and process for their preparation. EP0328150(A2) 1989.

Hartunian-Sowa SM, White PJ, Batres LV. Influence of monoglycerides of different chain lengths on texture and flavor of breads made with waxy cornstarch. Starch 1990; 42: 53-6.

Israelachvili J, Mitchell D, Ninham BW. Theory of self-assembly of hydrocarbon amphiphiles into micelles and bilayers. J Chem Soc Faraday Trans 1976; 2: 1525-68. Kabara JJ, Lie MSF. Antimicrobial lipids: natural and synthetic fatty acids and monoglycerides. Lipids 1977; 12: 753.

Kato N. Antimicrobial activity of fatty acids and their esters against a film-forming yeast in soy sauce. J Food Safety 1981; 3: 121-6.

Kamei T, Teraoka T, Hirano H. Antistatic acrylic polymer or polycarbonate laminate sheets. Japanese Patent JP 02276 636, 1990.

Koch K, Barthlott W. Superhydrophobic and superhydrophilic plant surfaces: an inspiration for biomimetic materials. Phil Trans $R$ Soc A 2009; 367: 1487-509.

Krog N. Interaction between water and surface active lipids in food systems. In: Duckworth RB (Ed.), Water Relation of Foods. London: Academic Press: 1975: 587.

Lachowicz A, Gahe G. Cyclo-carbonatecontg. ester(s) - by reaction of corresp. cyclo-carbonate-alcohol(s) with mono- or di-carboxylic acids in the presence of acid catalyst and solvent DE3937116(A1) 1991.
Leser ME, Sagalowicz L, Michel M, Watzke H. Self-assembly of polar food lipids. Adv Colloid Interface Sci 2006; 123-6: 125-36.

Lok C. M. Glyceride Esters. U.S. Patent 4,234,498, 1980.

Malkin T. The polymorphism of glycerides. Prog Chem Fats Other Lipids 1954; 2: 1-50.

Mc Intire RT. Fatty Acids in Industry; New York: Marcel Dekker, 1989.

Mouloungui Z. Voies inhabituelles de synthèse de composés oléophiles à partir des substrats végétaux solides (graines oléoprotéagineuses), liquides (huiles végétales et dérivés, glycérol) pour l'industrie chimique OCL 2004; 11: 425-35.

Mouloungui Z, Yoo JW, Gachen CA, Gaset A. Vermeersch G. Process for the preparation of glycerol carbonate from glycerol and a cyclic organic carbonate, especially ethylene or propylene carbonate EP0739888A1, 1996.

Mouloungui Z, Gauvrit C. Synthesis and influence of fatty acid esters on the foliar penetration of herbicides. Ind Crops Prod 1998; 8: 1-15.

Mouloungui Z, Rakotondrazafy V, Valentin R, Zebib B. Synthesis of glycerol 1-monooleate by condensation of oleic acid with glycidol catalyzed by anion-exchange resin in aqueous organic polymorphic system. Ind Eng Chem Res 2009; 48: 6949-56.

Mouloungui Z, Pelet S. Study of the acyl transfer reaction: Structure and properties of glycerol carbonate esters. Eur J Lipid Sci Technol 2001; 103: 216-22.

Mouloungui Z, Yoo JW. Glycerol catalytic carbonation: stoechiometric technology versus catalytic alternative for design of glycerol carbonate. Proceedings of Oleo and Speciality Chemicals Conference PIPOC 2007, 26-30 August 2007: 144-69.

O'Brien JL, Beavers EM. Process for preparing carbonatoalkyl acrylates and methacrylates. US patent 2979514, 1961.

Oki M, Nakanishi H. Conformations of the esters. V. Conformations of carbonates. Bull Chem Soc Jap 1973; 44: 3419-23.

Oehlenschläger J, Gercken G. Synthesis and mass spectrometry of 1-acyl and 3-acylsn-glycerol carbonates. Lipids 1978; 13: 557-62.

Queste S, Bauduin P, Touraud D, Kunz W, Aubry JM. Short chain glycerol 1-monoethers-a new class of green solvo-surfactants. Green Chem 2006; 8: 822-30.

Rieger M. Glyceryl stearate, chemistry and use. Cosmet Toiletries 1990; 105: 51-7.

Sein A, Verheij JA, Agterof WGM. Rheological characterization, crystallization, and gelation behavior of monoglyceride gels. J Colloid Interface Sci 2002; 249: 412-22. 
Silvestre F, Aubry JM, Benvegnu T, et al. Agroressources pour une chimie durable. Actual Chim 2010; 338-9: 28-40.

Tamura T. Preparation of higher fatty acid monoglycerides from fatty acids and glycidol. Japanese Patent JP 04182451, 1992.

Tanford C. The hydrophobic effect. NewYork: Wiley Interscience, 1980.

Valentin R, Alignan M, Giacinti G, Renaud FNR, Raymond B, Mouloungui Z. Pure short-chain glycerol fatty acid esters and glycerylic cyclocarbonic fatty acid esters as surface active and antimicrobial coagels protecting surfaces by promoting superhydrophilicity. I Colloid Interface Sci 2012; 365: 280-8.
Vieville C, Yoo JW, Pelet S, Mouloungui Z. Synthesis of glycerol carbonate by direct carbonatation of glycerol in supercritical $\mathrm{CO} 2$ in the presence of zeolites and ion exchange resins. Catal Lett 1998; 56: 245-7.

Vriet C, Mouloungui Z. Organic carbonates: a neglected family of compounds. Actual Chim 2008; 315: 19-27.

Werpy T, Petersen G (Eds.). Top value added chemicals from biomass 2004. PNNL, NREL, EERE (report 8674).

Yoo JW, Mouloungui Z. Catalytic carbonylation of glycerin by urea in the presence of zinc mesoporous system for the synthesis of glycerol carbonate. Stud Surf Sci Catal 2003; 146: 757-60.
Yoo JW, Mouloungui Z. The catalytic synthesis of the glycidol from the glycerol carbonate in presence of zeolite A. Stud Surf Sci Catal $2001 ; 135$.

Yoo JW, Mouloungui Z, Gaset A. Method for production an epoxide, in particular of glycidol, and installation for implementation. WO9840371(A1) 1998.

Zhu Y, Fournial AG, Molinier V, Azaroual N, Vermeersch G, Aubry JM. Self-association of short-chain nonionic amphiphiles in binary and ternary systems: comparison between the cleavable ethylene glycol monobutyrate and its ether counterparts. Langmuir 2009; 25: 761-8. 